\title{
Efecto de la fertilización nitrogenada y fosforada en el desarrollo y fenología de brotes de setos de Pinus radiata
}

\author{
Effect of nitrogen and phosphorus fertilization on Pinus radiata hedges \\ shoots phenology and growth \\ Andrés Hernández $C^{\mathrm{a}, \mathrm{b} *}$, Rafael Rubilar $\mathbf{P}^{\mathrm{a}}$ \\ *Correspondencia autor: ${ }^{\text {a }}$ Universidad de Concepción, Facultad de Ciencias Forestales, Departamento de Silvicultura, \\ Laboratorio de Suelos, Nutrición y Productividad Forestal Sustentable, Concepción, Chile. \\ b Instituto Forestal sede Concepción, Chile, jhernandez@infor.cl
}

\begin{abstract}
SUMMARY
The effect of nitrogen and phosphorus fertilization on shoot phenology and growth of Pinus radiata edges was evaluated during the productive growing season. A factorial design, considering nitrogen levels of 0, 100, 200 and $400 \mathrm{mg} \mathrm{L}^{-1}$ and phosphorus levels of 0, 50, 100, $150 \mathrm{mg} \mathrm{L}^{-1}$ as nutrient solutions, was applied considering water loss of 3 year old hedges grown in pots. Measurements of shoot elongation $( \pm 0.1 \mathrm{~cm})$ and diameter $( \pm 0.1 \mathrm{~mm})$ were made every $16 \pm 1$ days during five months for 9 shoots selected randomly. Total number of shoots qualifying for cutting production was evaluated at the end of the study for each selected plant. Nitrogen fertilization affected shoot elongation, but no response was observed to phosphorus fertilization. Nitrogen concentrations applied showed three groups (0, 100 - 200, and $400 \mathrm{mg} \mathrm{L}^{-1}$ of nitrogen) of magnitude of elongation responses. No differences were observed for shoot diameter among treatments. Elongation growth showed an exponential development that increased with nitrogen concentration in the nutrient solution. Nitrogen concentration also caused a linear increase in the number of shoots per plant. Nitrogen fertilization level also increased nitrogen concentration in shoots tissue which ranged from $13 \mathrm{~g} \mathrm{~kg}^{-1}$ with $0 \mathrm{mg} \mathrm{L}^{-1}$ of nitrogen to $24 \mathrm{~g} \mathrm{~kg}^{-1}$ with $400 \mathrm{mg} \mathrm{L}^{-1}$ of nitrogen. However, as the concentration of nitrogen increased in the growing medium, foliar potassium and calcium concentration decreased.
\end{abstract}

Key words: Pinus radiata, nitrogen, vegetative propagation, nutrition hedges, shoot elongation.

\section{RESUMEN}

Con el objetivo de determinar el efecto de la fertilización con nitrógeno y fósforo sobre el desarrollo y crecimiento fenológico de los brotes de setos de Pinus radiata, se estudió el desarrollo y fenología de tales brotes en setos sometidos a distintos niveles de fertilización nitrogenada $\left(0,100,200\right.$ y $\left.400 \mathrm{mg} \mathrm{L}^{-1}\right)$ en combinación factorial con fósforo $\left(0,50,100,150 \mathrm{mg} \mathrm{L}^{-1}\right)$. Se realizaron mediciones de elongación $( \pm 0,1 \mathrm{~cm})$ y diámetro $( \pm 0,1 \mathrm{~mm})$ de brotes cada $16 \pm 1$ días durante cinco meses. Los niveles de fertilización con nitrógeno afectaron la elongación de los brotes, no existiendo respuesta con fosforo. Para las concentraciones de nitrógeno evaluadas, se diferenciaron tres grupos (0, 100 - 200, y $400 \mathrm{mg} \mathrm{L}^{-1}$ de nitrógeno) desde el punto de vista de la magnitud de respuesta en crecimiento en elongación; en cambio, el diámetro de cuello no mostró diferencia en crecimiento. La tasa de crecimiento en elongación a partir de la fase de crecimiento exponencial aumentó a medida que aumentaban las concentraciones de nitrógeno en la solución nutritiva. El aumento en la concentración de nitrógeno provocó un incremento lineal en el número de brotes y en las concentraciones de nitrógeno en los tejidos, las cuales variaron desde $13 \mathrm{~g} \mathrm{~kg}^{-1}$ con $0 \mathrm{mg} \mathrm{L}^{-1}$ de nitrógeno hasta $24 \mathrm{~g} \mathrm{~kg}^{-1}$ con $400 \mathrm{mg} \mathrm{L}^{-1}$ de nitrógeno. A medida que incrementó la concentración de nitrógeno del medio de crecimiento disminuyó la concentración foliar de potasio y de calcio.

Palabras clave: Pinus radiata, nitrógeno, propagación vegetativa, nutrición de setos, elongación de brotes.

\section{INTRODUCCIÓN}

La propagación vegetativa de Pinus radiata D. Don se ha transformado en una herramienta vital para incorporar la ganancia genética en las nuevas plantaciones forestales realizadas con la especie. Se han desarrollado diversos programas de enraizamiento de estacas en diversas especies, así mismo, los productores de plantas continuamente han buscado maximizar la cantidad y calidad de las estacas producidas en los jardines de setos mediante el desarrollo de nuevos esquemas de manejo basados en regímenes de fertilización y riego específicos, de manera de optimizar el proceso de brotación de nuevas yemas desde los setos y la posterior elongación del brote formado.

Variados estudios con respecto al efecto de la fertilización, en la fenología de plantaciones en $P$. radiata sugieren que la elongación de los brotes responderá a diferencias en la calidad del sitio y niveles nutricionales de las plantas (Dougherty et al. 1994, Rubilar 2005). Se ha evidenciado que los patrones de crecimiento de los brotes difieren entre 
árboles adultos, juveniles y setos, ya que se sabe que especies del género Pinus presentan diferentes patrones fenológicos según su etapa de desarrollo (Dougherty et al. 1994).

En los programas de nutrición tanto el nitrógeno como el fósforo son considerados elementos esenciales para la producción de plantas. Reich y Shoettle (1988) sugieren que la eficiencia relativa del uso del nitrógeno en la fotosíntesis depende directamente de la disponibilidad del fósforo, induciendo en conjunto un incremento en el crecimiento de la planta. Rowe et al. (2002) verifican que la fertilización con nitrógeno en setos de Pinus taeda L. aumenta los niveles de carbohidratos, mejorando la producción de brotes y enraizamiento adventicio. Asimismo, estudios de fertilización muestran un incremento en el crecimiento, en respuesta al aumento de las concentraciones de nitrógeno en plántulas de Pinus elliotti Engelm. (Comerford y Fisher 1984).

Muchos estándares nutricionales publicados en la literatura han sido desarrollados para diversas especies (Dewald et al. 1992, Henry et al. 1992, Monsalve et al. 2009) producidas a través de semillas en viveros forestales, $\mathrm{y}$ enfocados en optimizar el crecimiento en altura y diámetro. Sin embargo, no existen estándares nutricionales documentados para producir y mejorar la calidad de los brotes en setos de $P$. radiata. Otras investigaciones han reportado que los esquemas de nutrición está enfocados en una rápida formación y producción de raíces y modificar la cantidad relativa de nitrógeno y carbohidratos, induciendo una influencia en el enraizamiento adventicio (Moe y Andersen 1988) y producción de brotes (Rowe et al. 2002).

Bajo estos antecedentes el objetivo de esta investigación es determinar el efecto de la fertilización con diferentes niveles de nitrógeno y fósforo suministrados a setos de $P$. radiata sobre el desarrollo y crecimiento fenológico de sus brotes, basado en la hipótesis que el aumento en las concentraciones de nitrógeno y fósforo en el medio de crecimiento provoca un aumento en el diámetro y elongación de los brotes de los setos.

\section{MÉTODOS}

Antecedentes generales. El estudio fue realizado en el vivero Proplantas Ltda., ubicado en la región del Biobío, Chile ( $36^{\circ} 37^{\prime} 23^{\prime \prime}$ S, $72^{\circ} 21^{\prime} 23^{\prime \prime}$ ) ). La zona presenta un clima mediterráneo templado cálido, con una temperatura media anual de $14{ }^{\circ} \mathrm{C}$, una máxima media del mes más cálido (enero) de $28,8^{\circ} \mathrm{C}$ y una mínima media del mes más frío (julio) de $3,5^{\circ} \mathrm{C}$.

Se utilizaron setos de tres años de edad de $P$. radiata, los cuales fueron originados a partir de semillas provenientes de huertos semilleros de la Corporación Nacional Forestal de Chile (CONAF), siendo establecidos en bolsas de polietileno de $1.000 \mathrm{~cm}^{3}$ con sustrato de compost de corteza de $P$. radiata en una granulometría de $74,3 \%$ menor a $3 \mathrm{~mm}, 20,7 \%$ entre 3 y $5 \mathrm{~mm}$ y 5,0 \% mayor a $5 \mathrm{~mm}$. En enero de 2009, fueron seleccionados aleatoriamente 144 setos, los cuales fueron sometidos a una poda de formación de copa a una altura de $25 \pm 1 \mathrm{~cm}$. El diseño experimental correspondió a bloques completos al azar con tres repeticiones, considerando un diseño factorial de cuatro concentraciones de nitrógeno $(0,100,200$ y $400 \mathrm{mg}$ $\left.\mathrm{L}^{-1}\right)$ y cuatro concentraciones de fósforo $(0,50,100,150$ $\left.m g \mathrm{~L}^{-1}\right)$. Las dosis de nitrógeno y fósforo se aplicaron en solución con el riego, manteniendo constante el resto de los macroelementos (potasio, calcio, magnesio y azufre). Todas las combinaciones de sales fueron balanceadas según el requerimiento de los tratamientos (cuadro 1).

La unidad experimental estuvo conformada por tres setos, donde fueron seleccionados en forma aleatoria nueve brotes. Cada 15 días \pm 1 , durante cinco meses (28 de enero hasta 25 de junio 2009) fueron realizadas mediciones de crecimiento en elongación $( \pm 0,1 \mathrm{~cm})$ y diámetro $( \pm 0,1 \mathrm{~mm})$. Al final de la temporada de crecimiento $(25 \mathrm{de}$ junio), cinco brotes terminales de $9 \mathrm{~cm}$ de longitud fueron colectados y secados a $65{ }^{\circ} \mathrm{C}$ hasta llegar a peso constante y molidos para análisis nutricionales. Para el análisis de nitrógeno se utilizó un analizador de nitrógeno y carbono NC 2100 (NC 2100 Soil, CE Instruments Ltd., Hindley Green, Wigan, UK), y en el caso de fósforo, potasio y calcio, se realizó una digestión en ácido nítrico mediante el método descrito por Jones y Case (1990). Las concentraciones de cada elemento se determinaron mediante un espectrómetro de emisión atómica con fuente de plasma acoplado inductivamente (ICP-2R, Varian Instruments).

El aporte de elementos nutritivos del agua de riego del vivero fue determinado mediante un análisis químico, incorporando este antecedente al balance de las soluciones

Cuadro 1. Fuentes utilizadas para los macroelementos en la fertirrigación de setos de $P$. radiata. Sources of macroelements used in the nutrient solution treatments applied to hedges.

\begin{tabular}{lll}
\hline $\begin{array}{l}\text { Elemento } \\
\text { nutritivo }\end{array}$ & $\begin{array}{l}\text { Concentraciones } \\
\text { evaluadas }\left(\mathrm{mg} \mathrm{L}^{-1}\right)\end{array}$ & \multicolumn{1}{c}{ Fuente } \\
\hline $\mathrm{N}$ & $0,100,200,400$ & $\mathrm{NaNO}_{3},\left(\mathrm{NH}_{4}\right)_{2} \mathrm{HPO}_{4}, \mathrm{CO}\left(\mathrm{NH}_{2}\right)_{2},\left(\mathrm{NH}_{4}\right)_{2} \mathrm{SO}_{4}$ \\
$\mathrm{P}$ & $0,50,100,150$ & $\left.\mathrm{KH}_{2} \mathrm{PO}_{4}, \mathrm{Ca}_{(} \mathrm{H}_{2} \mathrm{PO}_{4}\right)_{2}$ \\
$\mathrm{~K}$ & 100 & $\mathrm{~K}_{2} \mathrm{SO}_{4}, \mathrm{~K}_{2} \mathrm{CO}_{3}, \mathrm{KH}_{2} \mathrm{PO}_{4}$ \\
$\mathrm{Mg}$ & 40 & $\mathrm{MgSO}_{4}$ \\
$\mathrm{~S}$ & 60 & $\mathrm{MgSO}_{4}, \mathrm{~K}_{2} \mathrm{SO}_{4},\left(\mathrm{NH}_{4}\right)_{2} \mathrm{SO}_{4}$ \\
$\mathrm{Ca}$ & 80 & $\mathrm{CaCO}_{3}, \mathrm{Ca}\left(\mathrm{H}_{2} \mathrm{PO}_{4}\right)_{2}$ \\
\hline
\end{tabular}


generadas. Además, se verificó el pH del agua de riego, no siendo necesaria su modificación de acuerdo al valor objetivo cifrado de 6,5. Las preparaciones de las soluciones nutritivas fueron realizadas según la metodología propuesta por Landis (1989). Los tratamientos nutricionales fueron aplicados como soluciones nutritivas vía fertirrigación, considerando primero la adición de $1.000 \mathrm{~mL}$ de solución de nitrato de calcio $\mathrm{Ca}\left(\mathrm{NO}_{3}\right)_{2}$ y luego 1.000 $\mathrm{mL}$ de solución con los otros elementos a fin de evitar la precipitación del calcio con los sulfatos y fosfatos utilizados. La frecuencia de riego fue establecida considerando la pérdida del $20 \%$ del peso del contenedor de cada seto respecto a la capacidad de campo del sustrato, el cual fue monitoreado diariamente a través de una balanza digital de precisión de $\pm 1 \mathrm{~g}$. Una vez alcanzado este nivel, fueron regadas de forma manual añadiendo $1.000 \mathrm{~mL}$ de agua, obteniendo un riego a saturación. Cada 20 días se calibró el agua aprovechable producto del aumento en biomasa vegetal del seto.

Análisis, ajustes de curvas de elongación y crecimiento en diámetro, en brotes. Se realizaron ajustes de curvas de crecimiento para el desarrollo en diámetro y elongación de los brotes considerando tres modelos no lineales (cuadro 2). Los ajustes se efectuaron mediante técnicas de regresión no lineal. Como estadígrafo de comparación entre modelos se utilizó la raíz del cuadrado medio residual (RMSE). Los análisis fueron realizados utilizando el procedimiento PROC NLIN del software estadístico SAS.

El efecto de la nutrición sobre el modelo de regresión se evaluó mediante la prueba de suma extra de cuadrados realizada a través de la prueba $\mathrm{F}$ (Weisberg 1985), siendo útil para determinar el efecto de la nutrición en el modelo de regresión ajustado en forma independiente para cada concentración de nitrógeno y fósforo, en contraste con un ajuste promedio por grupos de cada concentraciones de elementos. El modelo complejo corresponde al ajuste por concentración individual de nitrógeno; mientras que el modelo simple, al ajuste promedio de un grupo de concentraciones.
Una vez seleccionados y agrupados los modelos no lineales, la elongación y el diámetro fueron estimados para periodos de 10 días. Los incrementos de crecimiento (\%) fueron determinados según Rubilar (2005).

Al final del período de crecimiento fueron realizados análisis de varianza (ANDEVA) para evaluar el efecto de las dosis de nitrógeno y fósforo en la elongación, crecimiento en diámetro, número de brotes producidos y los macronutrientes foliares. La homogeneidad de varianza fue evaluada mediante la prueba de Levene $(P<0,05)$. El supuesto de normalidad de los residuos se evaluó mediante la prueba de Shapiro-Wilks $(P<0,05)$. Para detectar diferencias significativas entre los tratamientos, se realizó la prueba de comparación múltiple Tukey, con un $95 \%$ de confianza.

\section{RESULTADOS}

Efecto de la nutrición en el crecimiento de los brotes. La elongación total de los brotes durante el período de evaluación (cinco meses) incrementó significativamente $(P \leq 0,01)$ a medida que aumentó la concentración de nitrógeno, variando desde $4,5 \mathrm{~cm}$ con $0 \mathrm{mg} \mathrm{L}^{-1}$ hasta $8,7 \mathrm{~cm}$ al fertilizar con $400 \mathrm{mg} \mathrm{L}^{-1}$ de nitrógeno, provocando un aumento de $93 \%$ en el crecimiento (figura 1A). Contrastantemente, el aumento de las concentraciones de fósforo en la solución nutritiva no presentó una respuesta en la elongación de los brotes $(P>0,05)$ (figura 1A).

El crecimiento en diámetro (figura 1B) de los brotes no fue afectado por las concentraciones de $0,100,200 \mathrm{y}$ $400 \mathrm{mg} \mathrm{L}^{-1}$ de nitrógeno o 0, 50, 100 y $150 \mathrm{mg} \mathrm{L}^{-1}$ de fósforo $(P>0,05)$.

Ajuste de curvas de elongación y crecimiento en diámetro de los brotes. Los modelos Weibull y Richards fueron los que presentaron menores errores de ajuste independiente de las concentraciones nutricionales (datos no presentados). Al no existir un efecto en el crecimiento en elongación y diámetro de los brotes, con las diversas concentraciones de fosforo empleadas $(0,50,100$ y $150 \mathrm{mg}$ $\mathrm{L}^{-1}$ ), se procedió a analizar solo el efecto del nitrógeno en

Cuadro 2. Modelos utilizados para ajustar curvas de elongación y crecimiento en diámetro de los brotes y su primera derivada para cálculos de incrementos.

Models used to fit elongation and diameter shoot growth and first derivative providing estimates of modeled shoot growth rates.

\begin{tabular}{|c|c|c|}
\hline Nombre del modelo & Ecuación & Primera derivada \\
\hline Logístico & $y=\frac{k}{1+b^{*} e^{-a^{* t}}}$ & $y=\frac{k^{*} b^{*} a^{*} e^{-a^{* t}}}{\left(1+b^{*} e^{-a^{* t}}\right)^{2}}$ \\
\hline Weibull & $\mathrm{y}=\mathrm{k}^{*}\left(1-\mathrm{e}^{-\mathrm{a}^{* \mathrm{~b}}}\right)$ & $y^{\prime}=k^{*} a^{*} b^{*} t^{b-1} * e^{-a^{*} t^{b}}$ \\
\hline Richards & $\mathrm{y}=\mathrm{k}^{*}\left(1-\mathrm{e}^{\left.-\mathrm{a}^{* \mathrm{t}}\right)^{\mathrm{n}}}\right.$ & $y=k^{*} a^{*} n^{*} b^{*} e^{-a^{* t}}\left(1-b^{*} e^{-a^{* t}}\right)^{n-1}$ \\
\hline
\end{tabular}

k, b, a = parámetros; $\mathrm{t}$ = tiempo. 

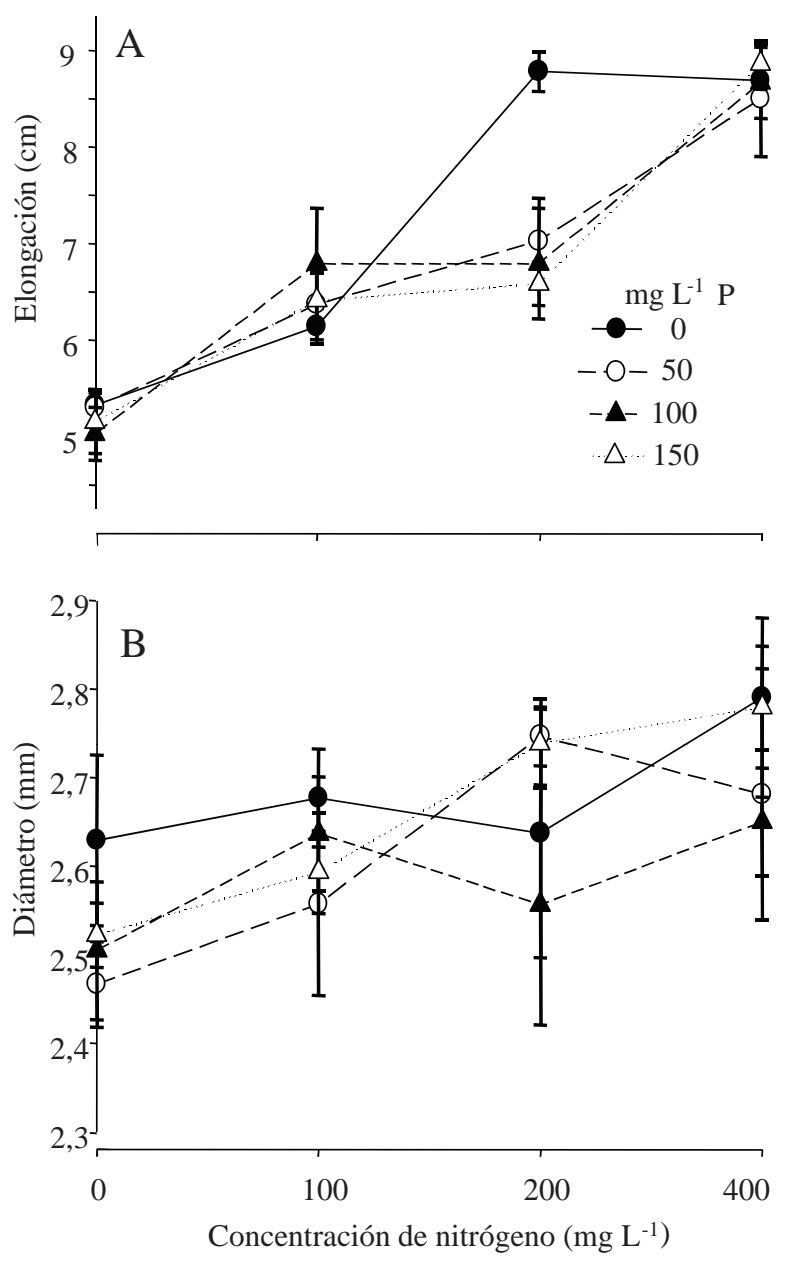

Figura 1. (A) Crecimiento en elongación (cm), (B) diámetro $(\mathrm{mm})$, de los brotes al término del estudio para los tratamientos aplicados de nitrógeno $\left(0,100,200\right.$ y $\left.400 \mathrm{mg} \mathrm{L}^{-1}\right)$ y fósforo $(0$, 50, 100 y $150 \mathrm{mg} \mathrm{L}^{-1}$ ) durante el experimento. La elongación de los brotes mostro un efecto significativo para los niveles de concentración de nitrógeno $(P \leq 0.01)$, pero no para fósforo. No se observó un efecto significativo para el diámetro de los brotes.

A) Elongation shoot growth (cm), and B) diameter shoot growth (mm) at the end of the study for nitrogen (0, 100, 200 and 400 $\left.\mathrm{mg} \mathrm{L}^{-1}\right)$ and phosphorus $\left(0,50,100\right.$ and $\left.150 \mathrm{mg} \mathrm{L}^{-1}\right)$ treatments applied during the experiment. Shoot elongation response showed significant effects for nitrogen concentration levels $(P \leq 0.01)$ but not for phosphorus. No significant effects of nitrogen or phosphorus treatments were observed for shoot diameter.

las curvas de crecimiento. Los tratamientos que recibieron equivalentes concentraciones de nitrógeno $\left(\mathrm{mg} \mathrm{L}^{-1}\right)$ mostraron un crecimiento similar en elongación durante el transcurso del tiempo, por ello se procedió agrupar por concentraciones de nitrógeno. Los grupos formados por 0 $\mathrm{mg} \mathrm{L}^{-1}$ nitrógeno $\left(\mathrm{F}=0,6<\mathrm{F}_{\alpha}={ }_{0,05 ; 9,96}=1,98\right), 100-200$ $\mathrm{mg} \mathrm{L}^{-1}$ de nitrógeno $\left(\mathrm{F}=1,56<\mathrm{F}_{\alpha=0,05 ; 9,96}=1,6\right)$ y $400 \mathrm{mg}$ $\mathrm{L}^{-1}$ de nitrógeno $\left(\mathrm{F}=1,52<\mathrm{F}_{\alpha=0,05 ; 9,96}=1,87\right)$ reducen en promedio entre $30 \%$ y $60 \%$ la variación residual de los modelos evaluados modificando los valores $\mathrm{y}$, en algunos casos, la significancia de los parámetros. Los modelos de regresión generados, formaron estimadores confiables con buenos indicadores de precisión, existiendo diferencias de crecimiento entre los grupos $\left(\mathrm{F}=58,4>\mathrm{F}_{\alpha=0,05 ; 6,423}=\right.$ 2,12 ), constatando una evidencia clara del efecto del nitrógeno sobre las funciones de crecimiento en elongación, evidenciado en los resultados obtenidos en la prueba de suma extra de cuadrados.

El diámetro no presentó una respuesta a las concentraciones de nitrógeno o fósforo, por lo cual sólo un modelo de regresión general se ajustó, el cual estuvo constituido por el total de los tratamientos ( $\mathrm{F}=1,40$ $\left.<\mathrm{F}_{\alpha=0,05 ; 45,384}=1,45\right)$. Con base en los resultados obtenidos en la prueba de suma extra de cuadrados no se evidenció un efecto de la fertilización en el crecimiento en diámetro para concentraciones de nitrógeno o fósforo, por tanto fue suficiente el uso de una función promedio para estimar el crecimiento en diámetro.

La elongación y el crecimiento en diámetro de los brotes presentaron un patrón de desarrollo correspondiente a una curva sigmoidal en el tiempo. La asíntota superior alcanzada para la elongación del brote fue de 8,0 , 6,8 y $5,3 \mathrm{~cm}$ para los grupos 400 de nitrógeno, $100-200$ de nitrógeno y 0 de nitrógeno en $\mathrm{mg} \mathrm{L}^{-1}$ (figura $2 \mathrm{~A}$ ) y de 2,6 mm para el diámetro (figura 2B). Los puntos de inflexión se alcanzaron a los $90 \pm 5$ días de 1,0, 0,8 y 0,7 mm (figura 2C) y en el día 78 de $0,34 \mathrm{~mm}$ para el diámetro (figura 2D), representando los puntos donde la aceleración del proceso cambia de signo pasando de una velocidad creciente a una decreciente.

Las curvas se caracterizaron por una primera fase de desarrollo lenta desde la poda de formación del seto hasta el brote de nuevas yemas ( 8 de enero al 7 de marzo) registrándose, condiciones promedio de temperaturas $20^{\circ} \mathrm{C}$, 10,5 horas de sol e intensidad de radiación de $28,5 \mathrm{MJ} \mathrm{m}^{-2}$. La segunda fase de la curva continúa con una aceleración en el crecimiento ( 7 de marzo al 5 de abril), a pesar de producirse una disminución en la temperatura $\left(16,4^{\circ} \mathrm{C}\right)$, horas de sol $(7,4)$ y radiación promedio $\left(16,5 \mathrm{MJ} \mathrm{m}^{-2}\right)$. Durante este período se realizó un mayor número de adiciones de fertilizantes lo que estaría generando las mayores tasas de crecimiento. A partir del día 110 (20 de abril) se evidenció la existencia de una clara diferencia en la elongación de $4,1,4,8$ y $5,4 \mathrm{~cm}$ que marca el grupo de $400 \mathrm{mg} \mathrm{L}^{-1}$ de nitrógeno sobre los grupos $0 \mathrm{mg} \mathrm{L}^{-1}$ de nitrógeno y 100 - 200 $\mathrm{mg} \mathrm{L}^{-1}$ de nitrógeno, respectivamente.

La elongación relativa (\%) presentó diferencias en el patrón de crecimiento entre los grupos. A los noventa días se presentó una inflexión para el grupo $0 \mathrm{mg} \mathrm{L}^{-1}$ de nitrógeno, y a los cien días para el grupo $100-200 \mathrm{mg} \mathrm{L}^{-1} \mathrm{de}$ nitrógeno y $400 \mathrm{mg} \mathrm{L}^{-1}$ de nitrógeno de 12,3, 11,6 y 12,3 $\%$ (figura $2 \mathrm{E}$ ), lo cual estaría indicando que la nutrición afectó el crecimiento y prolongó el período de elongación por un período de diez días.

Efecto de la nutrición en la producción de brotes. No existió un efecto en las regresiones ajustadas con las diferentes concentraciones de fosforo empleadas $(0,50,100$ y 150 

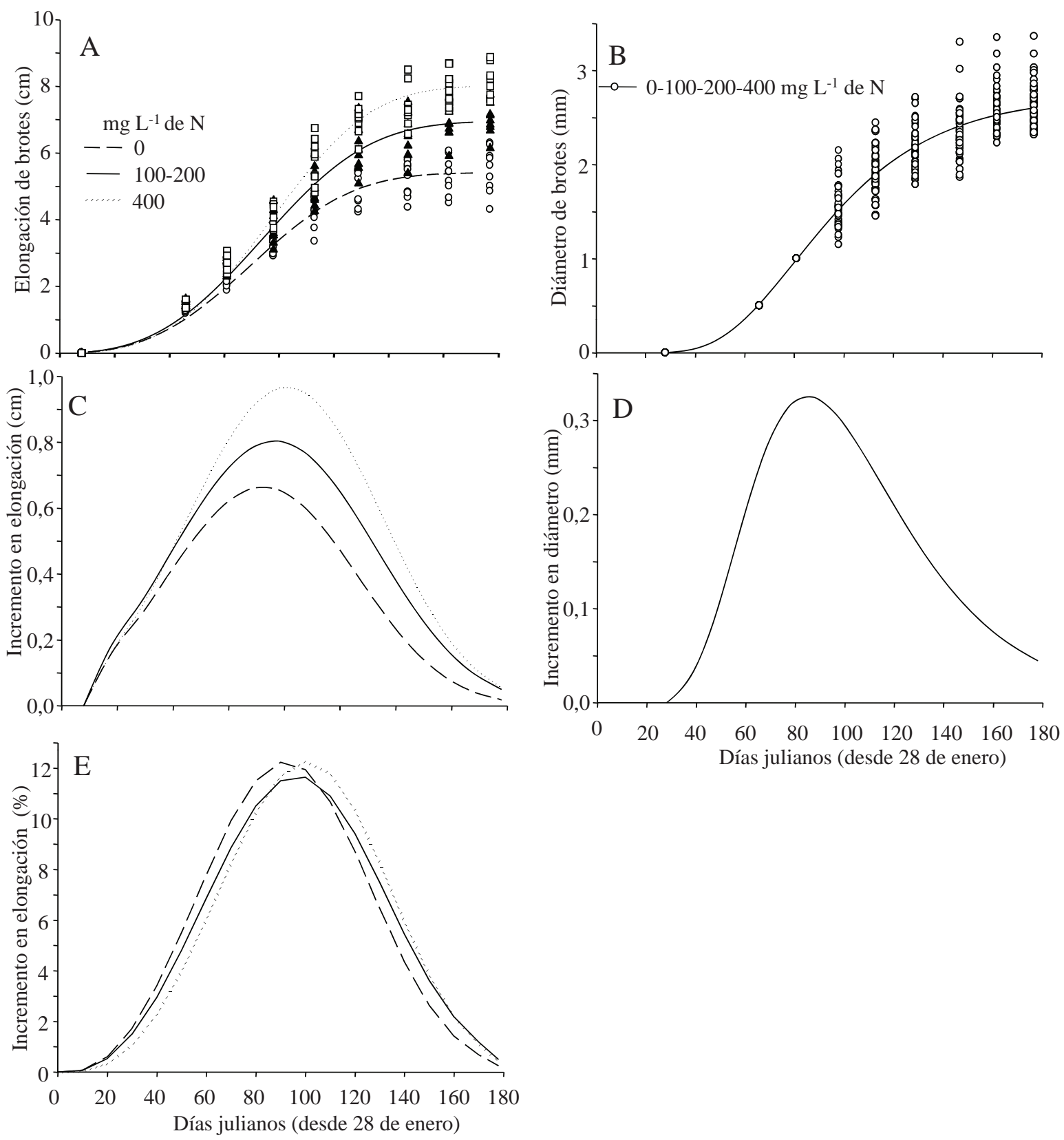

Figura 2. Modelos de crecimiento ajustados para desarrollo de brotes de setos de $P$. radiata creciendo en diferentes regímenes de nitrógeno. (A) Modelo de Weibull para elongación. (B) Modelo de Richards para crecimiento en diámetro. (C) Modelo de Weibull para el incremento en elongación (cm). (D) Modelo de Richards para el incremento en diámetro (mm). (E) Modelo de Weibull para el incremento en elongación (\%). Datos observados $(\circ, \boldsymbol{\Lambda}, \square)$ y predichos $(---, \ldots, \cdots \cdots)$.

Growth models adjusted for shoot development of P.radiata hedges grown under different nitrogen levels. A) Weibull model for shoot elongation, B) Richards model for shoot diameter, C) Weibull model for elongation growth rate (cm), D) Richards model for diameter growth rate (mm) and E) Weibull model for percent elongation development $(\%)$. Observed data $(\circ, \boldsymbol{\Lambda}, \square)$ and predicted $(---, \ldots, \cdots \cdots)$.

$m g \mathrm{~L}^{-1}$ ), presentando similares pendientes e intersecciones con el eje de las ordenas. Por ello, se procedió a realizar un ajuste promedio (datos no presentados).

Los tratamientos de mayor concentración de nitrógeno en la solución nutritiva mostraron el mayor número de brotes $\geq 5 \mathrm{~cm}$ en longitud (figura 3 ), presentando un efecto lineal positivo a medida que aumentaron las concentracio- nes de nitrógeno $\left(\mathrm{r}^{2}=0,84, P<0,0001\right)$.

Concentración de nutrientes en los tejidos. A medida que aumentaron las concentraciones de nitrógeno de $0 \mathrm{mg} \mathrm{L}^{-1}$ a $400 \mathrm{mg} \mathrm{L}^{-1}$ en la solución nutritiva aplicada a los setos, los niveles de nitrógeno $\left(\mathrm{g} \mathrm{kg}^{-1}\right)$ en los tejidos aumentaron desde $13 \mathrm{~g} \mathrm{~kg}^{-1}$ a $24 \mathrm{~g} \mathrm{~kg}^{-1}$ ajustándose una relación 


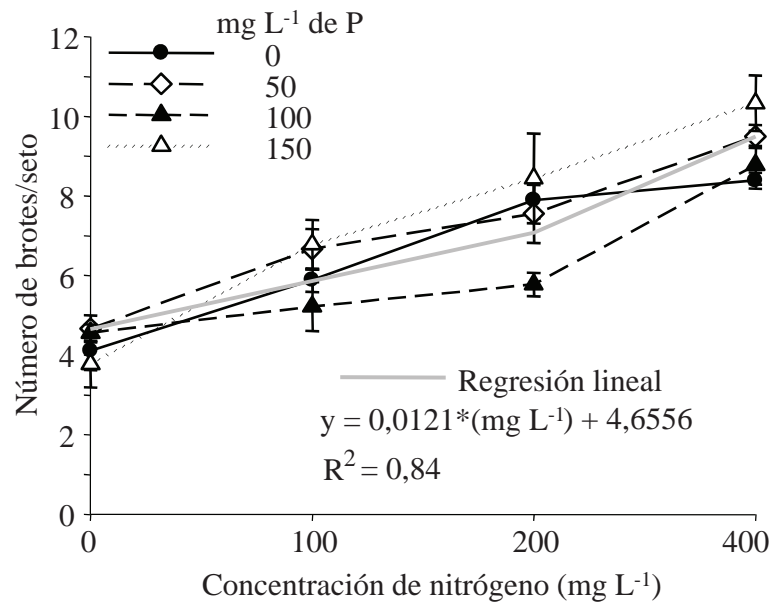

Figura 3. Número total de brotes generados para la propagación de estacas, para los diferentes tratamientos nutricionales. Las barras de error corresponden el error estándar de la media. Regresión lineal fue generada considerando el número de brotes y niveles de nitrógeno. Regresión lineal (-) representan la ecuación de regresión ( $\mathrm{n}=16, P \leq 0,01)$ promedio de todos los tratamientos de fósforo a través de los niveles de concentración de nitrógeno de los tratamientos.

Effect of nutrient treatments on total number of shoots produced that qualify for cuttings. Error bars correspond to mean standard error. A linear regression model was obtained considering number of shoots and nitrogen level. Regression line ( - ) represents a regression equation (n $=16, P \leq 0.01)$ averaging all phosphorus treatments across nitrogen concentrations level treatments.

lineal entre las concentraciones de nitrógeno en los tejidos y los niveles de nitrógeno y fósforo en la solución nutritiva $\left(r^{2}=0,81, P<0,0001\right)$ (figura 4A). Dicho comportamiento se mostró diferente con el nivel de potasio $\left(r^{2}=0,55\right.$, $P=0,0048)$ y calcio en planta $\left(r^{2}=0,74, P=0,0002\right)$, los cuales disminuyeron (figuras $4 \mathrm{C}$ y $4 \mathrm{D}$ ), conforme se incrementó la concentración de nitrógeno en el medio hasta los $200 \mathrm{mg} \mathrm{L}^{-1}$. Concentraciones más altas de nitrógeno no generaron una respuesta diferencial de ambos nutrientes en los tejidos. La fertilización no afectó a la concentración de fósforo (figura 4B) en los tejidos, registrándose valores de $2,1 \mathrm{~g} \mathrm{~kg}^{-1}$ para el tratamiento control $\left(0 \mathrm{mg} \mathrm{L}^{-1}\right.$ de nitrógeno y $0 \mathrm{mg} \mathrm{L}^{-1}$ de fósforo) y $2,3 \mathrm{~g} \mathrm{~kg}^{-1} \pm 3 \mathrm{~g} \mathrm{~kg}^{-1}$ en promedio para los tratamientos que contenían altos valores de fosforo (150 $\mathrm{mg} \mathrm{L}^{-1}$ de fósforo).

\section{DISCUSIÓN}

La elongación de los brotes muestra una relación directa con la concentración de nitrógeno en el medio de crecimiento, reflejando la alta sensibilidad de los setos de $P$. radiata a los cambios de concentración de nitrógeno en el medio. Esto sugiere deficiencias en elongación y desarrollo del brote al no fertilizar con nitrógeno. Dicho comportamiento es similar al observado en otras especies. Al comparar plantas de Prosopis chilensis Mol. sin fertilizar (control), con aquellas que fueron fertilizadas se verificó que la fertilización provoca un incremento en el crecimiento de las plantas entre $1.047 \%$ y $1.294 \%$ $(P<0,001)$ (Imo y Timmer 1992). Investigaciones realizadas en Pinus mariana Mill. determinaron que el crecimiento de las plantas en vivero aumenta significativamente $(P<0,001)$ desde un $257 \%$ hasta un $361 \%$ con la fertilización con nitrógeno. Según Dewald et al. (1992) el incremento en los niveles de nitrógeno en el follaje de Pinus elliotti, producto del aumento de las concentraciones de nitrógeno en la solución nutritiva, provoca un incremento en la biomasa total, traduciéndose en un aumento en el crecimiento de los brotes. Esto coincide con Elliot y White (1994) en plantas juveniles de Pinus resinosa Ait., corroborando los conceptos planteados por Landis (1989), quien señala que niveles elevados de nitrógeno promueven una rápida división y elongación celular. Salisbury y Ross (2000) plantean que el nitrógeno se encuentra presente en muchos compuestos esenciales, no sorprendiendo en absoluto que el crecimiento sea lento cuando existe una baja disponibilidad de nitrógeno. Sin embargo, el crecimiento en diámetro de los brotes no es afectado por la nutrición con nitrógeno o fósforo. Esto podría ser explicado probablemente dado el efecto de la alta densidad a la cual se encontraban establecidos los setos y al alto número de brotes existentes por seto (20 \pm 5 ), lo que provoca una competencia entre brotes de una misma rama. De esta manera, un mayor número de brotes estaría favoreciendo la elongación de la misma no así su diámetro.

Estudios en Pinus resinosa han evidenciado que la adición de fósforo no afecta la biomasa (Elliot y White 1994). Sin embargo, Chang (2003) indica que existe un mayor crecimiento en respuesta a una mayor disponibilidad de fósforo para Liquidambar styraciflua L. de un año de edad. El incremento en crecimiento en respuesta a una mayor disponibilidad de fósforo ha sido reportado para varias especies de árboles establecidos en campo o en condiciones artificiales (Chang 2003). Estas contradicciones se deben probablemente a la relación existente entre el fósforo y la intensidad de luz. Altas concentraciones de fósforo (516 mM) y bajas intensidades de radiación fostosintéticamente activa (120 - $\left.180 \mu \mathrm{mol} \mathrm{m}^{-2} \mathrm{~s}^{-1}\right)$, disminuyen las tasas de crecimiento relativo, existiendo un efecto del fósforo solo cuando ambos, luz y fósforo, se incrementan en plantas juveniles de Pinus pinaster Ait. (Cheaïb et al. 2005). Asimismo, se ha reportado que la biomasa en plantas de Pinus resinosa creciendo bajo alta luz $(>800 \mu \mathrm{mol}$ $\left.\mathrm{m}^{-2} \mathrm{~s}^{-1}\right)$ mostró cuatro a cinco veces más biomasa que en baja luz $\left(190 \mu \mathrm{mol} \mathrm{m}^{-2} \mathrm{~s}^{-1}\right)$.

El ajuste de los modelos de regresión mostró estimadores confiables para la elongación y el crecimiento en diámetro y los datos del estudio sugieren que la elongación de los brotes en $P$. radiata puede ser estimada con el modelo de Weibull. El ajuste de los datos en estudio concuerda con los datos de Sheffield et al. (2003), quienes estimaron el crecimiento fenológico de brotes en árboles adultos de 

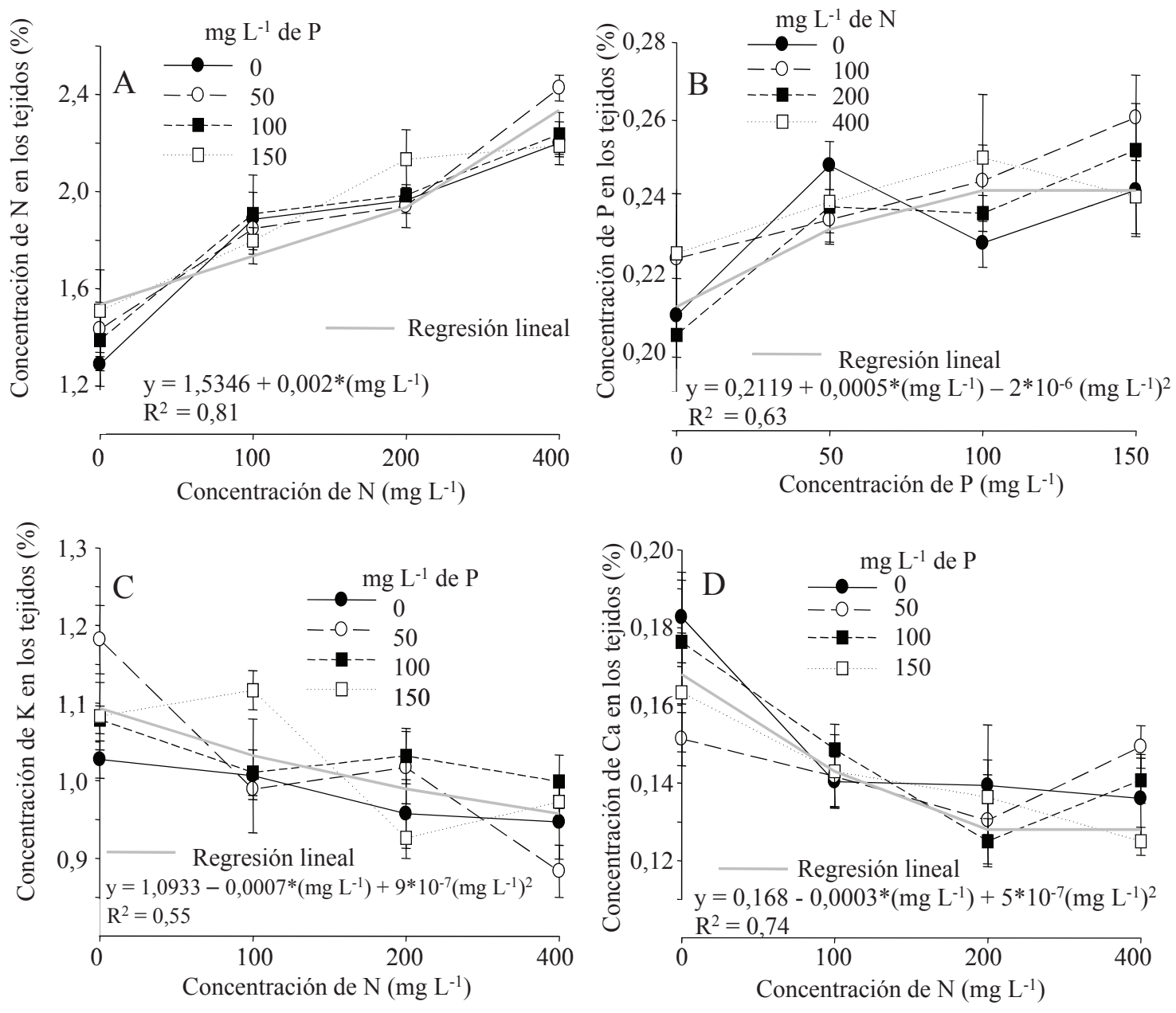

Figura 4. Concentraciones foliares obtenidas al final del estudio en brotes de setos de $P$. radiata para cada tratamiento evaluado. Las barras de error son los errores estándar de la media. Regresión lineal (-) representan la ecuación de regresión $(\mathrm{n}=16, P \leq 0,01)$ promedio de todos los tratamientos de fósforo a través de los niveles de concentración de nitrógeno de los tratamientos.

Shoot tissue nitrogen, phosphorus, potassium and calcium concentrations obtained at the end of the study for each evaluated treatment. Error bars are standard errors of the mean. A linear regressions was generated from averages of phosphorus treatments across nitrogen levels $(n=16$, $P \leq 0.01)$.

P. radiata con un modelo de Weibull. Un modelo similar fue establecido por Baltunis y Greenwood (1999) en brotes de Larix decidua Mill.

A medida que se incrementan las concentraciones de nitrógeno en la solución nutritiva, aumenta la elongación de los brotes durante la fase de crecimiento exponencial, la cual se extiende hasta el final de la estación de crecimiento. Las curvas se caracterizan por una primera fase lenta desde la formación del seto hasta la brotación ( 8 de enero al 7 de marzo), período en el cual se produce un aumento paulatino de la brotación, concordando con los datos de Cremer (1992) en arboles adultos de P. radiata. Las respuestas en crecimiento producto de la fertilización nitrogenada nunca son inmediatas (Monsalve et al. 2009), esto se ve reflejado en el estudio ya que las respuestas en crecimiento sólo acontecieron transcurrido 110 días de iniciado la fertilización, y al cabo de cinco meses de fertilización se verificaron diferencias significativas entre los grupos de 0 nitrógeno, 100 - $200 \mathrm{mg} \mathrm{L}^{-1}$ y $400 \mathrm{mg} \mathrm{L}^{-1}$ de nitrógeno. Asimismo, se verificó una modificación en la estructura de la copa, lo que según McCrady y Jokela (1998) estaría mejorando la intercepción de luz a través de una mejor masa de follaje y desarrollo fenológico de los brotes.

Los modelos de crecimiento evaluados presentan patrones clásicos de respuesta al gradiente nutricional establecido: (1) crecimiento deficiente de los brotes $0 \mathrm{mg} \mathrm{L}^{-1}$ de nitrógeno, (2) niveles óptimos de crecimiento con altas concentraciones de nitrógeno (400 $\mathrm{mg} \mathrm{L}^{-1}$ de nitrógeno), (3) no logrando alcanzar niveles de toxicidad en los tratamientos establecidos. Los resultados no concuerdan con Ingestad (1979), quien logra toxicidad en árboles juveniles de Pinus sylvestris L. con $400 \mathrm{mg} \mathrm{L}^{-1}$ de nitrógeno creciendo durante nueve meses en un sustrato de arena gruesa de cuarzo y en Pinus elliotti con $300 \mathrm{mg} \mathrm{L}^{-1}$ de nitrógeno (Dewald 
et al. 1992). Las distintas respuestas observadas pueden estar relacionadas a las tasas de elongación de cada especie, pero además al medio de crecimiento utilizado. La capacidad de intercambio catiónico para arena gruesa de cuarzo presenta valores muy bajos (Landis 1989), comparado con el medio de corteza del género Pinus que es capaz de retener una alta cantidad de nutrientes, balanceando la disponibilidad de nitrógeno en la solución del medio de crecimiento.

Las prácticas relacionadas al manejo nutricional de los setos son realizadas para optimizar el enraizamiento adventicio de los brotes producidos. Según Rowe et al (2002), el aumentar los niveles de nitrógeno a los setos de Pinus taeda fomenta un aumento en el número de brotes durante el periodo de primavera desde 22 brotes para 10 $\mathrm{mg} \mathrm{L}^{-1}$ de nitrógeno hasta 50 brotes para $55 \mathrm{mg} \mathrm{L}^{-1}$ de nitrógeno, pero las altas concentraciones de nitrógeno en los tejidos no necesariamente corresponden a un alto porcentaje de enraizamiento en brotes.

Los niveles de macronutrientes foliares mostraron, que en la medida que aumentaron las concentraciones de nitrógeno en la solución nutritiva se incrementaron las concentraciones de nitrógeno a nivel foliar, no importando la concentración de fósforo disuelto en el medio de crecimiento. Con el tratamiento de $400 \mathrm{mg} \mathrm{L}^{-1}$ de nitrógeno se obtiene la mayor concentración de nitrógeno a nivel foliar, sin embargo, desde los $100 \mathrm{mg} \mathrm{L}^{-1}$ de nitrógeno se pueden obtener niveles de nitrógeno que se encuentran en el rango recomendado como óptimo, según lo propuesto por Duryea y McClain (1984), para plantas de coníferas producidas en contenedor. Asimismo el aumento de las concentraciones de nitrógeno en la solución nutritiva provocó un incremento lineal en el crecimiento los brotes, no siendo mayormente relevante, debido que estacas de tercer orden no presentan similares respuesta rizogenicas que estacas de primer orden, debido probablemente a la variación en las concentraciones de nitrógeno foliar a lo largo del brote.

No existe información publicada de los niveles de nitrógeno y fosforo óptimos en setos de $P$. radiata, siendo estos de importancia para su posterior respuesta al enraizamiento de las estacas. Por otra parte, para los tratamientos sin nitrógeno las plantas no alcanzan el óptimo de nitrógeno foliar señalado por Duryea y McClain (1984), lo que fue observado por los síntomas de deficiencia asociados al follaje pequeño y de color amarillo-verdoso (Landis 1989). El potasio fue observado en niveles adecuados para todos los tratamientos (Duryea y McClain 1984). Sin embargo, la concentración de $400 \mathrm{mg} \mathrm{L}^{-1}$ de nitrógeno genera incrementos en crecimiento, en comparación con la concentración $200 \mathrm{mg} \mathrm{L}^{-1}$ de nitrógeno. Los resultados del estudio sugieren que aún no se habría alcanzado el nivel óptimo para nutrición nitrogenada en la fase de crecimiento rápido propuesta por (Landis 1989). La relación entre la concentración de nitrógeno foliar en respuesta a la aplicación de nitrógeno es generalmente asintótica. Similares resultados se han demostrado en pino oregón (Pseudotsuga menziesii (Mirb.) Franco) y en Juniperus virginiana L. (Henry et al. 1992). Sin embargo, niveles óptimos varían con la especie (Rowe et al. 2002). En setos de Pinus taeda, las concentraciones de nitrógeno en los tejidos aumentan con el incremento de las aplicaciones de nitrógeno hasta los $70 \mathrm{mg} \mathrm{L}^{-1}$ de nitrógeno, encontrando variaciones en los niveles de nitrógeno en el follaje, los que varían desde 13 $\mathrm{g} \mathrm{kg}^{-1}$ al no fertilizar con nitrógeno y $24 \mathrm{~g} \mathrm{~kg}^{-1}$ al fertilizar con $400 \mathrm{~mL} \mathrm{~L}^{-1}$ de nitrógeno (Rowe et al. 2002). La mayor concentración de nitrógeno en la planta permite la promoción de raíces, ácido nucleicos, síntesis de proteínas y un mayor metabolismo de carbohidratos. Rowe et al. (2002) encuentran que brotes obtenidos de setos de $P$. taeda fertilizados a tasas de $50 \mathrm{mg} \mathrm{L}^{-1}$ de nitrógeno presentan un mayor porcentaje de enraizamiento, esto sugiere que los brotes producidos en el presente estudio podrían presentar mayores niveles de enraizamiento y crecimiento posterior a las estacas y futuras plantas a viverizar.

$\mathrm{Al}$ aumentar la concentración de nitrógeno en la solución nutritiva se observó un aumento en los contenidos de nitrógeno y una disminución en los contenidos de calcio y potasio en los tejidos. Sin embargo, los niveles de potasio se encuentran en el rango recomendado como óptimo (Landis et al. 1989), en el caso del calcio se encuentra bajo el rango recomendado como óptimo, por lo cual se encontraría en un intervalo denominado necesidad óptima, debido a que no hubo una disminución del crecimiento en elongación de los brotes producto de esta deficiencia.

\section{CONCLUSIONES}

A medida que aumentan las concentraciones de nitrógeno proporcionadas al medio de crecimiento $(0,100,200$ y $400 \mathrm{mg} \mathrm{L}^{-1}$ ) se incrementa el crecimiento en elongación de los brotes, sin embargo, el crecimiento en diámetro no es afectado. Las concentraciones de fósforo empleadas $\left(0,50,100\right.$ y $\left.150 \mathrm{mg} \mathrm{L}^{-1}\right)$, no afectaron al crecimiento en elongación y diámetro de los brotes.

$\mathrm{Al}$ aumentar las concentraciones de nitrógeno en el medio de crecimiento incrementan los niveles de este elemento en los tejidos vegetales, lo que se traduce en un incremento en el número promedio de brotes producidos por cada seto. Por lo tanto, la fertilización nitrogenada de los setos tiene un efecto positivo en el desarrollo y crecimiento fenológico de los brotes para la producción de estacas.

\section{AGRADECIMIENTOS}

Este proyecto de investigación fue desarrollado gracias al apoyo del gerente general del vivero Proplantas Ltda. Sr. Leopoldo Quezada, la colaboración del personal técnico del vivero, el aporte del Laboratorio de la Cooperativa de Nutrición Forestal para el desarrollo de los análisis nutricionales y a las alumnas de la carrera de Ingeniería 
Forestal de la Universidad de Concepción. Srtas. Daniela García y Graciela Neira.

\section{REFERENCIAS}

Baltunis B, A Greenwood. 1999. Variation in lateral shoot elongation patterns and hybrid vigor in full-sib families and interspecific hybrids of larch. Tree Physiology 19 (2): 131136.

Comerford N, R Fisher. 1984. Using foliar analysis to classify nitrogen-deficient sites. Soil Science Society of America 48: 910-914.

Cheaïb A, A Mollier, S Thunot, C Lambort, S Pellerin, D Loustau. 2005. Interactive effects of phosphorus and light availability on early growth of maritime pine seedlings. Annals of Forest Science 62: 575-583.

Chang S. 2003. Seedling sweetgum (Liquidambar styraciflua L.) halfsib family response to $\mathrm{N}$ and $\mathrm{P}$ fertilization: growth, leaf area, net photosynthesis and nutrient uptake. Forest Ecology and Management 173: 281-291.

Cremer K. 1992. Relations between reproductive growth and vegetative growth of $P$. radiata. Forest Ecology and Management 52: 178-199.

Dewald L, T White, M Durya. 1992. Growth and phenology of seedlings of four contrasting slash pine families in ten nitrogen regimes. Tree Physiology 11: 255-269.

Duryea ML, K McClain. 1984. Altering seedling physiology to improve reforestation success. In Duryea ML, GN Brown eds. Seedling physiology and reforestation manual. Success I. Proceedings of the Physiology Working Group Technical Session. Oregon State University. Corvallis, Oregon. USA. p. 77-114.

Dougherty P, D Whitehead, J Vose. 1994. Environmental influences on the phenology of pine. Ecological Bolletins 43: 64-75.

Elliott K, A White. 1994. Effects of light, nitrogen, and phosphorus on red pine seedling growth and nutrient use efficiency. Forest Science 40(1): 47-50.

Henry P, F Blazich, L Hinesley. 1992. Nitrogen nutrition of containerized eastern redcedar. II. Influence of stock plant fertility on adventitious rooting of stem cuttings. Journal of the American Society for Horticultural Science 117: 568-570.

Ingestad T. 1979. Mineral nutrient requirements of Pinus sylvestris and Picea abies seedlings. Plant Physiology 45: 373-380.

Imo M, V Timmer. 1992. Nitrogen uptake of mesquite seedlings at conventional and exponential fertilization schedules. Soil Science Society of America Journal 56(3): 927-934.

Jones J, V Case. 1990. Sampling, handling and analyzing plant tissue samples. In Westerman RL Eds. Soil testing and plant analysis. Soil Science Society of America. Madison, Wisconsin, Estados Unidos. p. 389-428.

Landis TD, RW Tinus, SE McDonald, JP Barnett. 1989. Seedling Nutrition and Irrigation. Vol. 4. The Container Tree Nursery Manual. Agric. Handbook 674. Washington, DC: U.S. Department of Agriculture, Forest Service. 119 p.

Moe R, A Anderson. 1988. Stock plant environment and subsequent adventitious rooting. In Davis TD, BE Hayssig, N Sankhla. Adventitious Root Formation in Cuttings. Dioscorides Press, Portland, Oregon. p. 214-234.

Monsalve J, R Escobar, M Acevedo, M Sánchez, R Coopman. 2009. Efecto de la concentración de nitrógeno sobre atributos morfológicos, potencial de crecimiento radical y estatus nutricional en plantas de Eucalyptus globulus producidas a raíz cubierta. Bosque 30(2): 88-94.

McCrady R, E Jokela. 1998. Canopy dynamics, light interception, and radiation use efficiency of selected loblolly pine families. Forest Science 44: 64-72.

Rowe D, F Blazich, C Raper. 2002. Nitrogen nutrition of hedged stock plants of loblolly pine I. Tissue nitrogen concentrations and carbohydrate status. New Forest 24: 39-51.

Reich P, A Schoettle. 1988. Role of Phosphorus and Nitrogen in Photosynthetic and Whole Plant Carbon Gain and Nutrient Use Efficiency in Eastern White Pine. Oecologia 77(1): 25-33.

Rubilar R. 2005. Environmental constraints on growth phenology, leaf area display, and above and belowground biomass accumulation of Pinus radiata (D. Don) in Chile. Thesis Ph.D. Raleigh, Estados Unidos. Department of Forestry, North Carolina State University. 208 p.

Salisbury F, C Ross. 2000. Fisiología Vegetal. Madrid, España. Thomson Editores, Spain Paraninfo. 988 p.

Sheffield M, J Gagnon, S Jack, D McConville. 2003. Phenological patterns of mature longleaf pine (Pinus palustris Miller) under two different soil moisture regimes. Forest Ecology and Management 179: 157-167.

Weisgerber S. 1985. Applied Linear Regresion, Second edition. New York, USA. Wiley. 324 p. 
